\title{
INTER-RELAÇÕES ENTRE AS PROPRIEDADES FÍSICAS E OS COEFICIENTES DA CURVA DE RETENÇÃO DE ÁGUA DE UM LATOSSOLO SOB DIFERENTES SISTEMAS DE USO ${ }^{(1)}$
}

\author{
Jorge Luiz Machado $^{(2)}$, Cássio Antonio Tormena ${ }^{(3)}$, Jonez Fidalski ${ }^{(4)}$ \\ \& Carlos Alberto Scapim ${ }^{(3)}$
}

\begin{abstract}
RESUMO
O comportamento físico e hídrico do solo fundamenta as práticas de seu uso e manejo. $\mathrm{O}$ objetivo deste trabalho foi avaliar as inter-relações entre as propriedades físicas e os coeficientes da curva de retenção de água de um Latossolo Vermelho distrófico sob diferentes sistemas de uso. Em 2004, foram selecionadas três áreas contíguas utilizadas por mais de vinte anos com mata (nativa), pousio (Brachiaria decumbens) e cultivo (culturas anuais). Foram coletadas 25 amostras de solo com estrutura não deformada na camada de $0-0,15 \mathrm{~m}$ de profundidade, que foram utilizadas para determinação das curvas de retenção de água do solo. As curvas de retenção de água foram ajustadas pela equação de van Genuchten, obtendo-se os coeficientes $\theta \mathrm{s}, \theta \mathrm{r}, \boldsymbol{\alpha}$ e $n$. Também foram mensuradas as variáveis densidade do solo, teor de C orgânico e teor de argila do solo (grupo I). Essas variáveis e os coeficientes das curvas de retenção do solo (grupo II) foram submetidos às análises de correlações canônicas. Verificou-se que a intensificação do uso do solo (mata, pousio e cultivo) resultou em maiores valores de densidade do solo e na redução dos teores de $\mathrm{C}$ orgânico e argila. $\mathrm{O}$ primeiro par das variáveis canônicas indicou dependência de $\theta$ s em relação a densidade, e o segundo par distinguiu os sistemas de uso do solo verificado pela dependência de $\theta \mathrm{r}$ em relação aos teores de argila e de $\mathbf{C}$ orgânico. As modificações dos teores de $\mathbf{C}$ orgânico e da densidade do solo sugerem a degradação da qualidade física e hídrica do solo.
\end{abstract}

Termos de indexação: densidade do solo, C orgânico do solo, correlações canônicas, manejo do solo, qualidade do solo.

\footnotetext{
(1) Parte da Tese de Mestrado do primeiro autor apresentada ao Programa de Pós-Graduação em Agronomia da Universidade Estadual de Maringá - UEM. Recebido para publicação em junho de 2006 e aprovado em novembro de 2007.

${ }^{(2)}$ Doutorando do Programa de Pós-Graduação em Agronomia - Área de concentração Solos e Nutrição de Plantas - Universidade Estadual de Maringá - UEM. Av. Colombo 5790, CEP 87020-900 Maringá (PR). Bolsista da CAPES. E-mail: jormachado@hotmail.com

(3) Professor Associado do Departamento de Agronomia, UEM. Bolsista do CNPq. E-mail: catormena@uem.br; cascapim@uem.br

(4) Pesquisador do Instituto Agronômico do Paraná - IAPAR. Área de Solos, Caixa Postal 564, CEP 87701-970 Paranavaí (PR). Email: fidalski@iapar.br
} 


\title{
SUMMARY: INTER-RELATIONSHIPS BETWEEN PHYSICAL PROPERTIES AND THE COEFFICIENTS OF SOIL WATER RETENTION CURVE IN AN OXISOL UNDER DIFFERENT SOIL USE
}

\begin{abstract}
Practices of soil use and management are based on the physical and hydric soil behavior. Our objective was to evaluate the inter-relationships between physical properties and coefficients of soil water retention curves in an Oxisol under different soil use systems. In 2004, three contiguous areas were selected, covered for over 20 years by forest (native), Brachiaria decumbens (fallow) and annual crops (cultivation). Twenty-five undisturbed soil samples were collected at a depth of $0-0.15 \mathrm{~m}$ to determine the soil water retention curves. The curves were fitted by the van Genuchten equation and $\theta \mathrm{s}, \theta \mathrm{r} \theta$ and $\mathrm{n}$ coefficients were estimated. The soil bulk density, soil organic carbon and clay content were also measured. A canonical correlation analysis was performed with the variables soil organic carbon content, clay content and soil bulk density (group I) and the coefficients of the soil water retention curves (group II). It was verified that an intensified soil use (forest, fallow and tillage) resulted in higher soil bulk density and lower soil organic carbon and clay contents. The first pair of canonical variables indicated dependence of $\theta$ s from soil bulk density, while the second pair distinguished the soil use systems verified by the relation of $\theta r$ with the clay and organic carbon content. The modifications in the soil organic carbon content and soil bulk density indicate that there occurred physic and hydric degradation of the soil.
\end{abstract}

Index terms: soil bulk density, soil organic carbon, canonical correlations, soil management, soil quality.

\section{INTRODUÇÃO}

A curva de retenção de água é essencial em estudos de qualidade do solo com vistas a nortear as práticas de uso e o manejo sustentável dos sistemas de produção agrícola. Modificações na estrutura do solo associadas à compactação e à perda da estabilidade dos agregados alteram a distribuição do tamanho dos poros, bem como a retenção, o movimento e a disponibilidade de água no solo.

A curva de retenção da água do solo é descrita pelo teor de água $(\theta)$ e potencial mátrico $(\Psi)$, com decréscimo lento e contínuo dessas variáveis durante a drenagem do solo. O conteúdo de água retido em determinado $\Psi$ decorre da estrutura e da distribuição dos tamanhos de poros (Beutler et al., 2002). Os resultados de Rawls et al. (1991) mostram que, em elevados $\Psi$, a curva de retenção é influenciada por poros estruturais associados ao efeito da matéria orgânica na formação e na estabilidade da estrutura do solo. Em baixo $\Psi$, a composição granulométrica e a mineralogia do solo tornam-se mais importantes devido à superfície disponível para a adsorção de água (Gupta \& Larson, 1979). Dessa forma, a curva de retenção de água permite relações de dependência entre os coeficientes das equações matemáticas que a descrevem com diferentes propriedades do solo, como o teor de argila, o teor de C orgânico, a área superficial específica e a densidade do solo (van den Berg et al., 1997).

Correlações de Pearson entre propriedades físicas do solo e os coeficientes de equações descritivas da curva de retenção de água do solo foram realizadas por Gupta \& Larson (1979), Rawls et al. (1991, 2003), van den Berg et al. (1997) e Rasiah \& Aylmore (1998). Esta abordagem não permite estabelecer os efeitos diretos e indiretos de uma variável independente com variáveis dependentes. Uma maneira de contornar essas limitações seria a utilização de correlações canônicas. De acordo com Cruz et al. (2004), por meio dessa correlação é possível estimar a máxima correlação entre dois grupos de variáveis compostas por combinações lineares (inter-relações) das diferentes propriedades que os constituem.

A maioria dos estudos descreve as relações entre as variáveis do solo e os coeficientes da equação da curva de retenção numa ampla variação de solos. No entanto, sob um mesmo solo, os diferentes sistemas de uso e manejo podem promover modificações na sua estrutura e no comportamento físico-hídrico do solo. O objetivo deste trabalho foi avaliar as inter-relações entre as propriedades físicas e os coeficientes da curva de retenção de água de um Latossolo Vermelho distrófico sob diferentes sistemas de uso.

\section{MATERIAL E MÉTODOS}

A amostragem de solo foi realizada na Fazenda Experimental da Universidade Estadual de Maringá, localizada no município de Maringá, no noroeste do Estado do Paraná. O solo foi identificado como Latossolo Vermelho distrófico (Embrapa, 2006). Em 
2004, foram selecionadas três áreas contíguas com cerca de 5 ha, com uso do solo diferenciado nos últimos 20 anos, sendo (a) mata - floresta estacional semidecidual; (b) pousio - vegetado permanentemente com gramínea Brachiaria decumbens, manejada com três a quatro roçadas anuais, com trator e roçadeira; e (c) cultivo - culturas anuais com preparo convencional do solo por meio de aração na camada de $0-0,25 \mathrm{~m}$ de profundidade e gradagem leve.

Em cada área, foram selecionados, aleatoriamente, 25 pontos de amostragem do solo. Em cada ponto, na camada de $0-0,15 \mathrm{~m}$ de profundidade, foi retirado um bloco de solo (0,10 x 0,10 x 0,15 m) e, ao lado, uma amostra indeformada em anel metálico $(0,05 \mathrm{~m}$ de diâmetro e de altura). Os blocos de solo foram manualmente destorroados para a separação de agregados entre 2 e $4 \mathrm{~mm}$ de diâmetro, os quais foram utilizados para a dessorção de água em baixos $\Psi$.

Após devidamente preparadas, as amostras indeformadas foram saturadas por meio da elevação gradual de uma lâmina de água em bandejas, até atingir cerca de 2/3 da altura das amostras. A curva de retenção de água do solo foi determinada aplicando os $\Psi$ de -10, -20, -40, -60 e -80 hPa, utilizando uma mesa de tensão e os $\Psi$ de $-250,-500,-1.000$, $-2.000,-4.000$ e $-15.000 \mathrm{hPa}$, por meio de pressões aplicadas em placas porosas (Klute, 1986). Nas amostras indeformadas, aplicaram-se os $\Psi$ de -10, -20, $-40,-60,-80,-250,-500$ e $-1.000 \mathrm{hPa}$. Nos $\Psi$ de -2.000 , -4.000 e - $15.000 \mathrm{hPa}$, utilizaram-se agregados com diâmetro entre 2 e $4 \mathrm{~mm}$. A saturação dos agregados foi realizada diretamente na placa porosa, no interior da câmara de Richards, durante 24 h e por ascensão capilar. Após as amostras entrarem em equilíbrio no $\Psi$ aplicado, elas foram pesadas e colocadas em estufa $\mathrm{a} \pm 105^{\circ} \mathrm{C}$ para determinação da umidade à base de massa $(\theta g)$. A densidade do solo foi calculada conforme Blake \& Hartge (1986).

Após a secagem das amostras indeformadas, o solo foi retirado dos anéis, tamisado em peneira de $2 \mathrm{~mm}$ de diâmetro de malha. Nessas amostras, foram determinados o teor de C orgânico do solo, pelo método indireto da oxidação do C por via úmida (Walkley \& Black), e a granulometria, pelo método do hidrômetro (Embrapa, 1997).

A curva de retenção de água do solo foi descrita matematicamente por meio da função proposta por van Genuchten (1980), descrita como $\theta=\theta \mathrm{r}+\{(\theta \mathrm{s}-\theta \mathrm{r}) /$ $\left.\left[1+(\alpha \Psi)^{n}\right]^{m}\right\}$, em que $\theta$ é o conteúdo de água do solo $\left(\mathrm{kg} \mathrm{kg}^{-1}\right) ; \theta_{\mathrm{S}}$ é o conteúdo de água na saturação $\left(\mathrm{kg} \mathrm{kg}^{-1}\right)$; $\theta_{\mathrm{r}}$ é o conteúdo de água residual $\left(\mathrm{kg} \mathrm{kg}^{-1}\right) ; \Psi$ é o potencial mátrico (hPa); e $\alpha\left(\mathrm{hP} \mathrm{a}^{-1}\right)$ e $n$ são os coeficientes de ajuste da equação. Adotou-se a restrição $[m=1-(1 / n)]$ no ajuste da equação aos dados.

Os coeficientes $\theta \mathrm{s}, \theta \mathrm{r}, \alpha$ e $n$ da equação de van Genuchten (1980) foram estimados pelo método dos quadrados mínimos não lineares, utilizando-se a rotina "PROC NLIN" (SAS, 1999). A comparação das médias das variáveis densidade do solo, teor de $\mathrm{C}$ orgânico e teor de argila do solo foi feita adotando o intervalo de confiança da média de $95 \%(p=0,05)$, conforme Morettin \& Bussab (2003).

O teste de multicolinearidade e as análises de correlações canônicas foram realizados pelo software GENES (Cruz, 2001) em acordo com as recomendações de Cruz \& Carneiro (2003) e Cruz et al. (2004), entre o grupo das propriedades de solo (grupo I: teor de C orgânico do solo, teor de argila e densidade do solo) e o grupo dos coeficientes da equação de van Genuchten (grupo II: $\theta \mathrm{s}, \theta \mathrm{r}, \alpha$ e $n$ ). A multicolinearidade das matrizes foi fraca, com número de condição $(0,07 \leq \mathrm{NC} \leq 2,78)$ e fator de inflação da variância $(1,85 \leq \mathrm{VIF} \leq 8,64)$ para os três sistemas de uso.

Antes de submeter os dados às análises de correlações canônicas no software GENES (Cruz, 2001), realizaram-se previamente testes de normalidade dos dados das variáveis $(n=25)$, imprescindíveis para modelos matemáticos lineares (Vieira, 1999). Foi adotado o grau de confiança de 0,01 para o teste de normalidade Shapiro-Wilk, correspondendo ao menor grau de confiança utilizado para os coeficientes das correlações canônicas. Os teores de $\mathrm{C}$ orgânico do solo para o sistema de uso do solo/cultivo e os teores de argila para os sistemas de uso do solo/mata não apresentaram distribuição normal dos dados originais, respectivamente, com probabilidades de 0,0072 e 0,0091 pelo teste Shapiro-Wilk, utilizandose o procedimento "PROC UNIVARIATE" (SAS, 1999; Schlotzhauer \& Littell, 1997). Após transformações arco seno da raiz dos teores de C orgânico do solo dividido por 10 e logaritmo natural dos teores de argila, obteve-se distribuição normal de 0,0633 e 0,0197, respectivamente. As outras variáveis apresentaram distribuição normal dos dados originais, com probabilidades de 0,0197 a 0,9226 pelo teste ShapiroWilk. Desse modo, as duas variáveis transformadas e as demais variáveis foram submetidas às análises de variância, atendendo o pressuposto de distribuição normal para todas as variáveis (Schlotzhauer \& Littell, 1997; Vieira, 1999).

Para os conjuntos de 25 dados de cada uma das variáveis e sistema de uso do solo, foram calculados os intervalos de confiança da média:

$$
\mathrm{IC}=\overline{\mathrm{X}} \pm \underset{[(\mathrm{n}-1),(1-\alpha / 2)]}{\mathrm{s}} \frac{\mathrm{s}}{\sqrt{\mathrm{n}}}
$$

em que IC é o intervalo de confiança da média; $\bar{x}$ é a média aritmética; t é o valor da distribuição normal tabulado, obtido pelo número de dados (n) menos um grau de liberdade (n-1) e o grau de confiança para $95 \%(\alpha=0,05)$; e s é o desvio-padrão das amostras (Schlotzhauer \& Littell, 1997).

\section{RESULTADOS E DISCUSSÃO}

O teor médio de $\mathrm{C}$ orgânico do solo para o sistema de uso do solo/cultivo foi significativamente menor que 
o do solo/mata e do pousio (Quadro 1), fato também observado nestes solos por Cardoso et al. (1992) e Marun (1996) e em outros solos por Klein \& Libardi (2002) e Silva et al. (2005). A redução do teor de C orgânico no solo cultivado foi de 22 e $31 \%$, respectivamente, em relação ao pousio e à mata, inferiores aos verificados por Lal (1994). A redução dos teores de $\mathrm{C}$ orgânico nos solos cultivados deve-se ao menor retorno e à maior taxa de decomposição de material orgânico (Sanchez, 1981).

A intensificação do uso do solo de mata para pousio e cultivo proporcionou incremento significativo da densidade do solo (Quadro 1), também constatado neste solo por Araujo et al. (2004) e Marun (1996). O incremento na densidade caracteriza a compactação do solo sob cultivo e pousio, considerando-se como referência a densidade do solo entre 1,36 e 1,40 $\mathrm{Mg} \mathrm{m}^{-3}$ sob mata para a mesma classe de solo (Embrapa, 1984). A redução nos teores de matéria orgânica implica maior suscetibilidade à compactação do solo (Thomas et al., 1996), justificando o acréscimo de densidade do solo sob pousio e cultivo. Esse acréscimo na densidade do solo implica conseqüente redução da qualidade física e estrutural do solo verificada por Araujo et al. (2004) nesse solo.

O teor de argila da camada superficial do solo foi reduzido no solo sob cultivo (Quadro 1). De acordo com Cardoso et al. (1992), o uso intensivo desses solos com lavouras anuais reduz a espessura do horizonte superficial e os teores de matéria orgânica e, por conseguinte, aumenta o grau de dispersão da argila. A redução dos teores de argila da camada arável do solo (Quadro 1) pode ser devida à dispersão das partículas de argila e à remoção pela erosão com permanência seletiva das partículas de areia.

A equação de van Genuchten (1980) explicou mais de $99 \%$ da retenção de água do solo $\left(R^{2}>0,99\right)$, com valores de $\mathrm{F}$ altamente significativos $(\mathrm{p}<0,01)$ para todas as amostras. Os coeficientes $\theta \mathrm{s}, \theta \mathrm{r}, \alpha$ e $n$ foram estatisticamente significativos, pois estes não apresentaram valores nulos em seus limites de confiança, conforme recomendam Glantz \& Slinker (1990).

Houve similaridade dos coeficientes $\alpha$ e $n$ para os solos sob cultivo e pousio (Quadro 2). Os valores médios de $\theta \mathrm{r}$ e $\theta$ s foram estatisticamente superiores no solo sob mata, condizentes com os menores valores de densidade do solo e com os maiores teores de C orgânico. Esses resultados corroboram aqueles citados por Silva et al. (2005), que verificaram ampla distribuição de poros nos solos que não tiveram estresses físico e mecânico resultantes do uso e manejo. O maior valor de $n$ foi obtido no solo sob mata, indicando maior retenção de água devido à maior porosidade total e ampla variação de tamanho de poros (van Genuchten \& Nielsen, 1985).

Os sistemas de uso do solo alteraram a forma das curvas de retenção de água no solo (Figura 1). O solo sob mata apresentou maior retenção de água entre os $\Psi$ de -10 a $-100 \mathrm{hPa}$ e de -1.000 a $-15.000 \mathrm{hPa}$. Contrariamente, sob pousio, verificou-se ligeira superioridade de retenção de água entre -100 e $1.000 \mathrm{hPa}$, atribuída às diferenças dos valores de $n$ resultante da maior freqüência de poros com menores

Quadro 1. Análise descritiva e distribuição normal Shapiro-Wilk (W e $p<W)$ para as variáveis teor de carbono orgânico, densidade do solo e teor de argila nos sistemas de uso do solo sob cultivo (culturas anuais), pousio (Brachiaria decumbens) e mata (nativa)

\begin{tabular}{|c|c|c|c|c|c|c|c|}
\hline Sistema de uso do solo & Mínimo & Média ${ }^{(1)}$ & $\operatorname{ICM}^{(2)}$ & Máximo & $\mathrm{CV}(\%)^{(3)}$ & W & $\mathbf{p}<\mathrm{W}$ \\
\hline & \multicolumn{7}{|c|}{ Teor de C orgânico do solo $\left(\mathrm{g} \mathrm{kg}^{-1}\right)$} \\
\hline Cultivo (culturas anuais) & 4,35 & $7,41 \mathrm{~b}$ & 0,81 & 9,87 & 26,51 & 0,8808 & 0,0072 \\
\hline Pousio (Brachiaria decumbens) & 5,14 & $9,85 \mathrm{a}$ & 0,86 & 13,30 & 21,27 & 0,9635 & 0,4890 \\
\hline \multirow[t]{2}{*}{ Mata (nativa) } & 6,72 & $11,12 \mathrm{a}$ & 1,08 & 17,59 & 23,44 & 0,9502 & 0,2529 \\
\hline & \multicolumn{7}{|c|}{ Densidade do solo $\left(\mathrm{Mg} \mathrm{m}^{-3}\right)$} \\
\hline Cultivo (culturas anuais) & 1,67 & $1,75 \mathrm{a}$ & 0,02 & 1,84 & 2,51 & 0,9624 & 0,4642 \\
\hline Pousio (Brachiaria decumbens) & 1,67 & $1,71 \mathrm{~b}$ & 0,01 & 1,76 & 1,87 & 0,9057 & 0,0244 \\
\hline \multirow[t]{2}{*}{ Mata (nativa) } & 1,26 & $1,41 \mathrm{c}$ & 0,03 & 1,52 & 4,39 & 0,9675 & 0,5821 \\
\hline & \multicolumn{7}{|c|}{ Teor de argila $\left(\mathrm{g} \mathrm{kg}^{-1}\right)$} \\
\hline Cultivo (culturas anuais) & 140,00 & $185,60 \mathrm{~b}$ & 11,56 & 240,00 & 15,09 & 0,9457 & 0,2006 \\
\hline Pousio (Brachiaria decumbens) & 180,00 & $207,20 \mathrm{a}$ & 7,86 & 250,00 & 9,19 & 0,9441 & 0,1920 \\
\hline Mata (nativa) & 170,00 & $210,00 \mathrm{a}$ & 10,72 & 250,00 & 12,37 & 0,8858 & 0,0091 \\
\hline
\end{tabular}

(1) Médias seguidas da mesma letra na coluna para a mesma variável não diferem estatisticamente pelo intervalo de confiança da média de $95 \%(p=0,05) .{ }^{(2)}$ Intervalo de confiança da média de $95 \%(p=0,05) .{ }^{(3)}$ Coeficiente de variação. 
Quadro 2. Análise descritiva e distribuição normal Shapiro-Wilk (W e $p<W$ ) para os coeficientes da curva de retenção de água nos sistemas de uso do solo sob cultivo (culturas anuais), pousio (Brachiaria decumbens) e mata (nativa)

\begin{tabular}{|c|c|c|c|c|c|c|c|}
\hline Sistema de uso do solo & Mínimo & Média $^{(1)}$ & $\operatorname{ICM}^{(2)}$ & Máximo & $\operatorname{CV}(\%)^{(3)}$ & $\mathbf{w}$ & $\mathrm{p}<\mathrm{W}$ \\
\hline & & & & $\alpha\left(\mathrm{hPa}^{-1}\right)$ & & & \\
\hline Cultivo (culturas anuais) & 0,006 & $0,015 b$ & 0,002 & 0,029 & 33,15 & 0,9573 & 0,3641 \\
\hline Pousio (Brachiaria decumbens) & 0,003 & $0,013 b$ & 0,002 & 0,019 & 34,79 & 0,9589 & 0,3925 \\
\hline \multirow[t]{2}{*}{ Mata (nativa) } & 0,016 & $0,026 \mathrm{a}$ & 0,002 & 0,040 & 21,32 & 0,9557 & 0,3353 \\
\hline & \multicolumn{7}{|c|}{$\theta \mathrm{s}\left(\mathrm{kg} \mathrm{kg}^{-1}\right)$} \\
\hline Cultivo (culturas anuais) & 0,149 & $0,177 \mathrm{c}$ & 0,007 & 0,217 & 9,53 & 0,9747 & 0,7641 \\
\hline Pousio (Brachiaria decumbens) & 0,166 & $0,191 \mathrm{~b}$ & 0,006 & 0,216 & 7,75 & 0,9486 & 0,2337 \\
\hline \multirow[t]{2}{*}{ Mata (nativa) } & 0,258 & $0,330 \mathrm{a}$ & 0,014 & 0,423 & 10,19 & 0,9462 & 0,2052 \\
\hline & \multicolumn{7}{|c|}{$\theta \mathrm{r}\left(\mathrm{kg} \mathrm{kg}^{-1}\right)$} \\
\hline Cultivo (culturas anuais) & 0,045 & $0,065 \mathrm{c}$ & 0,004 & 0,087 & 13,98 & 0,9598 & 0,4115 \\
\hline Pousio (Brachiaria decumbens) & 0,057 & $0,076 \mathrm{~b}$ & 0,003 & 0,084 & 8,60 & 0,9189 & 0,0483 \\
\hline \multirow[t]{2}{*}{ Mata (nativa) } & 0,073 & $0,094 \mathrm{a}$ & 0,004 & 0,116 & 10,31 & 0,9821 & 0,9226 \\
\hline & & & & $n$ & & & \\
\hline Cultivo (culturas anuais) & 1,4147 & $1,675 \mathrm{~b}$ & 0,063 & 2,068 & 9,10 & 0,9556 & 0,3331 \\
\hline Pousio (Brachiaria decumbens) & 1,4055 & $1,676 \mathrm{~b}$ & 0,071 & 2,004 & 10,23 & 0,9602 & 0,4179 \\
\hline Mata (nativa) & 1,7818 & $2,244 \mathrm{a}$ & 0,119 & 2,695 & 12,81 & 0,9482 & 0,2287 \\
\hline
\end{tabular}

(1) Médias seguidas da mesma letra na coluna para o mesmo coeficiente não diferem estatisticamente pelo intervalo de confiança da média de $95 \%(p=0,05) .{ }^{(2)}$ Intervalo de confiança da média de $95 \%(p=0,05) .{ }^{(3)}$ Coeficiente de variação.

tamanhos nesta faixa de $\Psi$. As curvas de retenção de água nos solos sob pousio apresentou maior retenção de água no solo, comparada ao solo/cultivo. O aumento na densidade do solo promoveu modificações na distribuição de poros nos solos sob cultivo e pousio, principalmente naqueles poros que retêm água em $\Psi$ maiores que - $100 \mathrm{hPa}$, evidenciado pela forte redução de $\theta$ s e $n$ nestes solos (Quadros 1 e 2; Figura 1). Essas modificações nas características de retenção de água resultam em mudanças na qualidade física dos solos (Dexter, 2004).

As correlações canônicas explicaram 0,90, 0,92 e 0,93 das correlações entre as variáveis dos grupos I e II do primeiro par canônico $(\mathrm{p}<0,01)$ para os solos sob pousio, cultivo e mata (Quadro 3). As correlações canônicas entre as variáveis dos grupos I e II explicaram causa e efeito da dependência negativa de $\theta$ s com a densidade do solo, confirmando a importância da estrutura do solo na sua maior retenção de água. A densidade do solo influencia tanto a porosidade total como a distribuição dos poros de maior diâmetro, modificando as características de retenção de água do solo na faixa de $\Psi$ de -10 a $-100 \mathrm{hPa}$ (Figura 1), de acordo com Rasiah \& Aylmore (1998).

O segundo par canônico das variáveis dos grupos I e II apresentaram coeficientes de correlação de 0,64,

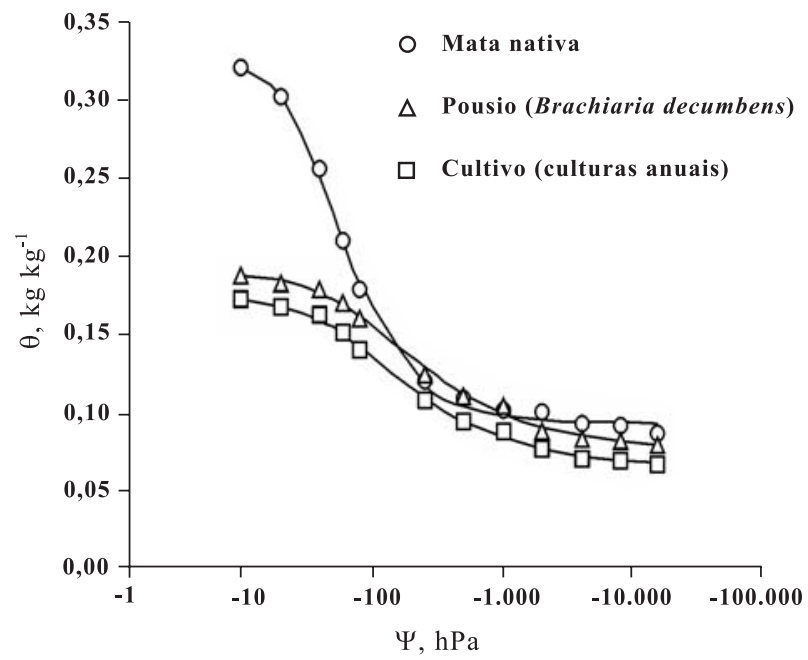

Figura 1. Curvas de retenção de água no solo para os sistemas de uso do solo sob cultivo (culturas anuais), pousio (Brachiaria decumbens) e mata (nativa).

$0,72$ e 0,72 ( $p<0,05, p<0,10$ e $p<0,05)$, respectivamente, para os sistemas de uso do solo sob cultivo, pousio e mata (Quadro 3). A correlação positiva dos teores de C orgânico do solo com o $\theta \mathrm{r}$ no 
Quadro 3. Correlações canônicas e pares canônicos $\left(\lambda_{n}\right)$ para os sistemas de uso de solo sob cultivo (culturas anuais), pousio (Brachiaria decumbens) e mata (nativa), estimadas entre os componentes primários, para as variáveis teores de carbono orgânico do solo, teores de argila e densidade do solo (grupo I), e secundários (grupo II), para os coeficientes da equação de van Genuchten $(\theta \mathrm{s}, \theta \mathrm{r}, \alpha$ e $n$ )

\begin{tabular}{|c|c|c|c|c|c|c|c|c|c|}
\hline \multirow[t]{2}{*}{ Variável ou coeficiente } & \multicolumn{3}{|c|}{ Cultivo (culturas anuais) } & \multicolumn{3}{|c|}{ Pousio (Brachiaria decumbens) } & \multicolumn{3}{|c|}{ Mata nativa } \\
\hline & $\lambda_{1}$ & $\lambda_{2}$ & $\lambda_{3}$ & $\lambda_{1}$ & $\lambda_{2}$ & $\lambda_{3}$ & $\lambda_{1}$ & $\lambda_{2}$ & $\lambda_{3}$ \\
\hline \multicolumn{10}{|l|}{ Grupo I } \\
\hline Teor de carbono orgânico & $-0,24$ & 0,52 & $-0,82$ & $-0,46$ & $-0,55$ & 0,69 & 0,11 & $-0,69$ & 0,71 \\
\hline Teor de argila & $-0,02$ & 0,42 & 0,91 & 0,20 & 0,98 & 0,05 & $-0,47$ & 0,87 & 0,14 \\
\hline Densidade do solo & 0,95 & 0,32 & 0,07 & 0,90 & $-0,24$ & 0,38 & 0,95 & 0,32 & $-0,07$ \\
\hline \multicolumn{10}{|l|}{ Grupo II } \\
\hline$\alpha$ & $-0,39$ & $-0,23$ & 0,57 & $-0,46$ & $-0,09$ & $-0,82$ & $-0,64$ & 0,39 & 0,16 \\
\hline$\theta \mathrm{s}$ & $-0,99$ & $-0,07$ & 0,14 & $-0,95$ & 0,15 & $-0,19$ & $-0,89$ & $-0,35$ & 0,29 \\
\hline$\theta \mathrm{r}$ & $-0,32$ & 0,74 & $-0,15$ & 0,02 & 0,75 & 0,42 & $-0,56$ & 0,46 & 0,18 \\
\hline$n$ & $-0,38$ & 0,16 & $-0,73$ & $-0,20$ & 0,01 & 0,79 & $-0,26$ & 0,20 & $-0,76$ \\
\hline$D c^{(1)}$ & 0,92 & 0,64 & 0,55 & 0,90 & 0,72 & 0,26 & 0,93 & 0,72 & 0,31 \\
\hline Significância ${ }^{(2)}$ & $* * *$ & $* *$ & * & $* * *$ & * & $\mathrm{ns}$ & $* * *$ & $* *$ & $\mathrm{~ns}$ \\
\hline
\end{tabular}

sistema de uso do solo cultivo demonstra a importância da matéria orgânica para a retenção de água, em solo cultivado, atribuída à alta superfície específica da matéria orgânica (Felton \& Ali, 1992; Olness \& Archer, 2005). Verificou-se dependência significativa de $\theta$ r com a interação dos teores de argila e de $\mathrm{C}$ orgânico do solo nos solos sob pousio e mata. A magnitude dos coeficientes dos teores de argila (0,98 e 0,87, respectivamente, para pousio e mata) em relação aos teores de $\mathrm{C}$ orgânico do solo (-0,55 e -0,69, respectivamente, para pousio e mata) revela que $\theta \mathrm{r}$ foi dependente simultaneamente da combinação de maiores teores de argila e menores teores de C orgânico do solo. As inter-relações antagônicas entre os teores de C orgânico do solo e os teores de argila são atribuídas à maior eficiência do $\mathrm{C}$ orgânico sobre $\theta \mathrm{r}$ em solos arenosos (Rawls et al., 2003). Essa afirmação é respaldada neste estudo pelos valores estatisticamente maiores dos teores de argila nos sistemas de uso do solo sob mata e pousio em relação ao cultivo (Quadro 1). As complexas interações entre estas variáveis também foram verificadas por Olness \& Archer (2005).

$\mathrm{O}$ solo sob cultivo diferenciou-se do solo sob pousio e mata por ter apresentado o terceiro par canônico com coeficientes de correlações canônicas de 0,55 $(\mathrm{p}<0,10)$ entre as variáveis dos grupos I e II (Quadro 3). O terceiro par canônico revelou que o coeficiente $n$ foi dependente da interação entre os teores de $\mathrm{C}$ orgânico e os teores de argila do solo. Tais resultados reiteram o efeito do C orgânico em $\theta$ r nos solos arenosos, a exemplo dos resultados obtidos por Rawls et al. (2003).
A contribuição das análises de correlações canônicas entre as propriedades do solo e os coeficientes da curva de retenção de água do solo é ter indicado que o uso desses solos deve ser norteado pela redução da densidade e a manutenção ou acréscimo de C orgânico do solo.

\section{CONCLUSÕES}

1. A intensificação do uso do solo (mata, pousio e cultivo) resultou em maiores valores de densidade do solo e na redução dos teores de C orgânico e de argila.

2. O primeiro par das variáveis canônicas indicou dependência de $\theta$ s em relação à densidade, e o segundo par distinguiu os sistemas de uso do solo verificado pela dependência de $\theta \mathrm{r}$ em relação aos teores de argila e de C orgânico do solo.

3. As modificações nos teores de C orgânico do solo e da densidade do solo explicam as mudanças negativas na curva de retenção de água dos solos sob pousio e cultivo, caracterizando o comprometimento da qualidade física e hídrica do solo.

\section{LITERATURA CITADA}

ARAUJO, M.A.; TORMENA, C.A. \& SILVA, A.P. Propriedades físicas de um Latossolo Vermelho distrófico cultivado e sob mata nativa. R. Bras. Ci. Solo, 28:337-345, 2004. 
BEUTLER, A.N.; CENTURION, J.F.; SOUZA, Z.M.; ANDRIOLI, I. \& ROQUE, C.G. Retenção de água em dois tipos de Latossolos sob diferentes usos. R. Bras. Ci. Solo, 26:829-834, 2002.

BLAKE, G.R. \& HARTGE, K.H. Bulk density. In: KLUTE, A., ed. Methods of soil analysis: Physical and mineralogical methods. 2.ed. Madison, American Society of Agronomy, 1986. p.363-375.

CARDoso, A.; POTTER, R.O. \& DEDECEK, A. Estudo comparativo da degradação de solos pelo uso agrícola no noroeste do Paraná. Pesq. Agropec. Bras., 27:349-353, 1992.

CRUZ, C.D. Programa GENES - versão windows. Versão 2005.0.0. Viçosa, MG, Universidade Federal de Viçosa, 2001. 642p.

CRUZ, C.D. \& CARNEIRO, P.C.S. Modelos biométricos aplicados ao melhoramento genético. Viçosa, MG, Universidade Federal de Viçosa, 2003. v.2. 585p.

CRUZ, C.D.; REGAZZI, A.J. \& CARNEIRO, P.C.S. Modelos biométricos aplicados ao melhoramento genético. 3.ed. Viçosa, MG, Universidade Federal de Viçosa, 2004. 480p.

DEXTER, A.R. Soil physical quality: Part I. Theory, effects of soil texture, density and organic matter and effects on root growth. Geoderma, 120:201-214, 2004.

EMPRESA BRASILEIRA DE PESQUISA AGROPECUÁRIA EMBRAPA. Manual de métodos de análise de solo. Rio de Janeiro, Centro Nacional de Pesquisa de Solos, 1997. 212p. (Embrapa-CNPS. Documentos, 1)

EMPRESA BRASILEIRA DE PESQUISA AGROPECUÁRIA EMBRAPA. Serviço Nacional de Levantamento e Conservação de Solos. Levantamento de reconhecimento dos solos do Estado do Paraná. Londrina, EmbrapaSNLCS/SUDESUL/IAPAR, 1984. v.1. p.135-215. (Embrapa-SNLCS. Boletim de Pesquisa, 27; IAPAR. Boletim Técnico, 16)

EMPRESA BRASILEIRA DE PESQUISA AGROPECUÁRIA EMBRAPA. Sistema brasileiro de classificação de solos. 2.ed. Rio de Janeiro, 2006. 306p.

FELTON, G.K. \& ALI, M. Hydraulic parameter response to incorporated organic matter in the B-horizon. Am. Soc. Agric. Eng., 35:1153-1160, 1992

GLANTZ, S.A. \& SLINKER, B.K. Primer of applied regression and analysis of variance. New York, McGraw-Hill, 1990. $777 \mathrm{p}$.

GUPTA, S.C. \& LARSON, W.E. Estimating soil water retention characteristics from particle size distribution, organic matter percent, and bulk density. Water Res. Res., 15:16331635,1979

KLEIN, V.A. \& LIBARDI, P.L. Densidade e distribuição do diâmetro dos poros de um Latossolo Vermelho sob diferentes sistemas de uso e manejo. R. Bras. Ci. Solo, $26: 857-867,2002$.
KLUTE, A. Water retention: Laboratory methods. In: KLUTE, A., ed. Methods of soil analysis. Physical and mineralogical methods. Madison, American Society of Agronomy, 1986. p.635-660

LAL, R. Methods and guidelines for assessing sustainable use of soil and water resources in the tropics. Washington, Soil Management Support Services, 1994. 78p. (SMSS. Technical Monograph, 21)

MARUN, F. Propriedades físicas e biológicas de um Latossolo Vermelho-Escuro do arenito Caiuá sob pastagem e culturas anuais. Pesq. Agropec. Bras., 31:593-597, 1996.

MORETTIN, P.A. \& BUSSAB, W.O. Inferência para várias populações. In: MORETTIN, P.A. \& BUSSAB, W.O, eds. Estatística básica. 5.ed. São Paulo, Saraiva, 2003. p.410435.

OLNESS, A. \& ARCHER, D. Effect of organic carbon on available water in soil. Soil Sci., 170:90-101, 2005.

RASIAH, V. \& AYLMORE, L.A.G. Sensitivity of selected water retention functions to compaction and inherent soil properties. Aust. J. Soil Res., 36:317-326, 1998.

RAWLS, W.J.; GISH, T.J. \& BRAKENSIEK, D.L. Estimating soil water retention from soil physical properties and characteristics. Adv. Soil Sci., 16:213-234, 1991.

RAWLS, W.J.; PACHEPSKY, Y.A.; RITCHIE, J.C.; SOBECKI, T.M. \& BLOODWORT, H. Effect of soil carbon on soil water retention. Geoderma, 116:61-76, 2003.

SANCHEZ, P.A. Suelos del trópico - Características y manejo. San José, Costa Rica, Instituto Interamericano de Cooperacion para la Agricultura, 1981. 645p.

SAS Institute. Statistical Analysis System Institute. SAS/STAT Procedure guide for personal computers. Version 5. Cary, 1999.

SCHLOTZHAUER, S.R. \& LITTELL, R.C. SAS ${ }^{\circledR}$ System for elementary statistical analysis. 2.ed. Cary, SAS Institute, 1997. 456p.

SILVA, A.J.N.; CABEDA, M.S.V. \& LIMA, F.W.F. Efeito de sistemas de uso e manejo nas propriedades físico-hídricas de um Argissolo Amarelo de tabuleiro costeiro. R. Bras. Ci. Solo, 29:833-842, 2005.

THOMAS, G.W.; HAZLER, G.R. \& BLEVINS, R.L. The effects of organic matter and tillage on maximum compactability of soils using the proctor test. Soil Sci., 161:502-508, 1996.

van den BERG, M.; KLAMT, E.; van REEUWIJK, L.P. \& SOMBROEK, W.G. Pedotransfer functions for the estimation of moisture retention characteristics of Ferralsols and related soils. Geoderma, 78:161-180, 1997.

van GENUCHTEN, M.T. A closed-form equation for predicting the hydraulic conductivity of unsaturated soils. Soil Sci. Soc. Am. J., 44:892-898, 1980. 
van GENUCHTEN, M.T.\& NIELSEN, D.R. On describing and predicting the hydraulic properties of unsatured soils. Ann. Geophys., 3:615-628, 1985.
VIEIRA, S. Pressuposições básicas. In: VIEIRA, S. Estatística experimental. 2.ed. São Paulo, Atlas, 1999. p.133-147. 